\title{
Light pionic atoms perspectives for precision experiments
}

\author{
D. Gotta \\ Institut für Kernphysik, Forschungszentrum Jülich, \\ D-52425 Jülich, Germany
}

\begin{abstract}
During the last decades high-precision spectroscopy of exotic-atom X-rays profited in particular in the case of pions from the increasing number of stopped particles provided by the cyclotron trap at the accelerator facility of the Paul-Scherrer-Institut (PSI) together with modern detector concepts like charge-coupled devices (CCDs) and crystal spectrometers. Presently, priority is given to the study of the strong-interaction effects in the most elementary system - pionic hydrogen. However, the systems with two or more nucleons are as fundamental for the development of a theoretical description of hadronic matter. Furthermore, the de-excitation of exotic atoms involves a variety of atomic processes, which become accessible in detail due to the high resolution achievable with crystal spectrometers, e. g., parallel transitions, line splittings, broadenings and intensity distributions. In addition, first successful attempts for a microscopic description of the atomic cascade are available now, which should be subject to stringent tests both for atoms and molecules.
\end{abstract}

\section{Introduction}

Exotic atoms constitute a laboratory allowing the study of fundamental particle-particle interactions, properties of stable and unstable particles as well as hadronic and electromagnetic interacting multi-body systems. In the case of hadrons the elementary systems formed with hydrogen isotopes are of particular interest. Measurements of the scattering length of the antiproton-nucleon, antiproton-deuteron and the corresponding systems with negatively charged pions have been performed the high-flux facilities LEAR at CERN and the meson factory at the Paul-Scherrer-Institut (PSI) [1].

Though high-precision experiments on pionic atoms using crystal spectrometers were started soon after the advent of the meson factories a substantial increase in particle flux and the ability to stop beams quantitatively even in gaseous targets had to be achieved to exploit the potentialities of diffraction techniques. Whenever a major progress in precison 


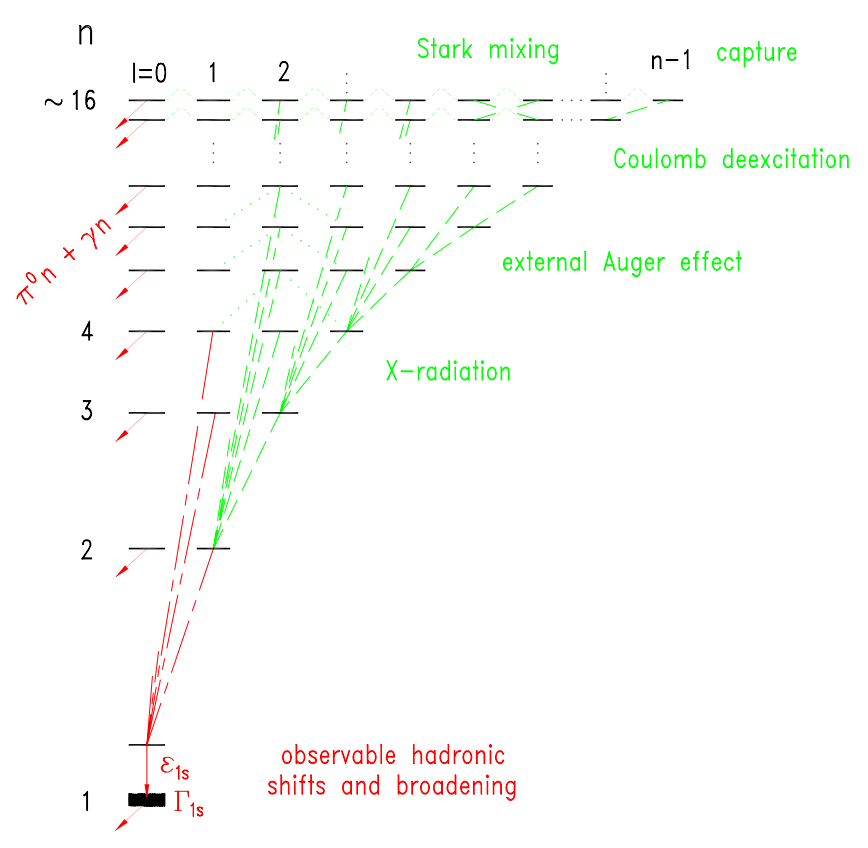

Figure 1: Atomic cascade in pionic hydrogen.

was achieved it turned out that a more refined knowledge of the atomic de-excitation is essential to determine the strong-interaction effects (Fig. 1). Consequently, as a matter on its own, cascade physics had to reach a higher level of sophistication $[2,3]$.

During the last decade the precision determination of the charged pion mass [4] and the strong-interaction effects in pionic hydrogen [5] was resumed. In the course of these experiments additional results and new perspectives for ultimate-precision X-ray spectroscopy arose. Topics outlined in this paper are:

- effects in pionic deuterium and the helium isotopes

- scaling of strong-interaction effects from high-lying atomic p states,

- strong-interaction hyperfine interaction in spin $\geq 1$ nuclei,

- test of spin zero particle bound-state QED (Klein-Gordon equation),

- measurements of transitions from the series limit and parallel transitions being sensitive to capture and de-excitation from high-lying states, and

- study of the interaction of the pion with the remnants of the electron shell. 


\section{Strong-interaction}

\section{$2.1 A \leq 4$ nuclei}

For pionic hydrogen a systematic study of the density dependence of line energy and width in combination with the measurement of three different transitions yielded an unambiguous extraction of the pure hadronic parameters. By means of a forthcoming high-statistics measurements of the $\pi H$ line width, the envisaged accuracy of $\leq 2 \%$ will allow a precise determination of the $\pi N$ scattering lengths $a^{+}$and $a^{-}$within the framework of chiral perturbation theory $(\chi \mathrm{PT})$. The accuracy of the theoretical prediction is reported to be $1 \%$ and $3 \%$ for $a^{+}$and $a^{-}$, respectively (for details see $[6]$ ).

In the case of pionic deuterium, additional aspects enter the theoretical description. At first, the construction of the $\pi d$ scattering length from the elementary $\pi N$ scattering lengths has to be accomplished facing dominating multi-body effects [7]. Secondly, absorption and re-emission of pions on the nucleon pair constitute an important contribution both to $\pi N N$ physics (with external pions) and the nuclear binding force (no external pions). The corresponding low-energy constant $\left(f_{0}\right.$ problem) appearing in effective field theory approaches is at present barely known [8]. Hence, within $\chi \mathrm{PT}$ calculations a determination of $a^{+}$and $a^{-}$is limited to about $10 \%$. Given the fact, that $a^{+}$and $a^{-}$can be reliably extracted from the $\pi H$ measurements, high-precision $\pi D$ data allow important tests, e. g., (i) limitation of the range of $f_{0}$ and comparison to theoretical approaches determining its value and (ii) extrapolation of the $N N \rightarrow d \pi$ cross section to threshold, which is directly related to the $\pi D$ ground-state broadening.

From the experimental side, in $\pi D$ the extraction of the strong-interaction shift and width is not proven to be as reliable as for $\pi H$. Calculations considering molecular formation $\pi D+D_{2} \rightarrow[(\pi d d) d]$ ee predict, that in $\pi D$ radiative de excitation out of molecular states is as important as Auger emission and even dominate in $\pi T[9,10]$. Such a de-excitation leads to satellite structures of (not known) intensity distribution. (i) No systematic study of the cascade has been performed yet to exclude molecular effects on the transition energy and (ii) the statistics of the two measurements performed does not allow to determine $\Gamma_{1 s}$ precisely $[11,12]$ (Fig. 2 and Tab. 1). The improvement of peakto-background ratio, increased detector size, and precise knowledge of the spectrometer response (Fig. 4) as developed for the hydrogen experiment are easily applicable to the $\pi D$ case.

Of principle interest is a comparison of the tri-nucleon systems $T$ and ${ }^{3} \mathrm{He}$. A measurement on the $1 \%$ level for the shift also gives access to the elementary $\pi N$ scattering lengths $a^{+}$and $a^{-}$and tests the approach of the nuclear wave functions used [13].

In view of the precison of shift and width of the helium isotopes, a remeasurements is desirable. Here, in addition to the ground-state effects, from the intensity balance of the $\mathrm{L}$ series and the $K \alpha$ transition, the $2 p$-state broadening is obtained. Furthermore, the isotope effect in the hadronic broadening should reflect the dominance of two-nucleon absorption at rest $[14,15]$. By using a crystal spectrometer, an improvement in accuracy of one order of magnitude is achievable. 

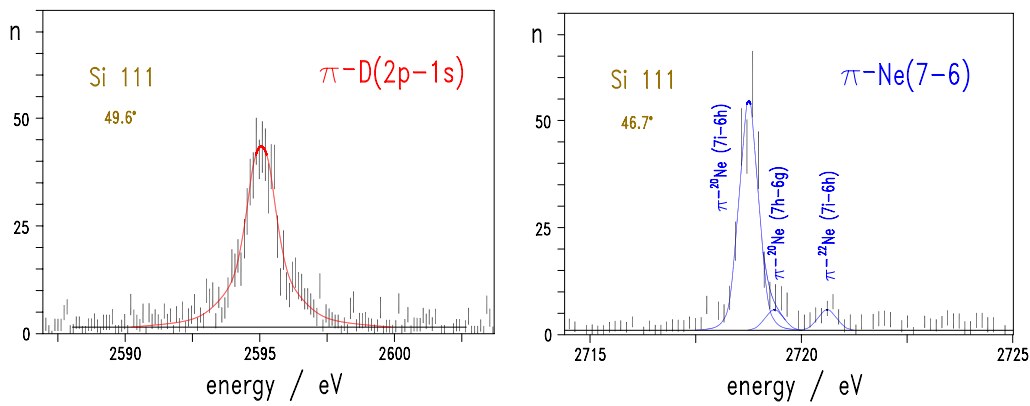

Figure 2: Line shape of the $\pi D(2 p-1 s)$ transition and corresponding spectrometer response function as determined from the $\pi N e(7 i-6 h)$ line.

Table 1: Strong-interaction effects in $A \leq 4$ nuclei. For the pure QED $\pi H(3 p-1 s)$ transition energy the most recent calculation yields $E_{3 p-1 s}^{Q E D}=2878.822 \mathrm{eV}[16]$. The experimental value is $E_{3 p-1 s}^{e x p}=2885.928 \pm 0.013 \mathrm{eV}[18]$.

\begin{tabular}{ccccc}
\hline & $\epsilon_{1 s}$ & $\Gamma_{1 s}$ & $\Gamma_{2 p}$ & \\
& $/ \mathrm{eV}$ & $/ \mathrm{eV}$ & $/ \mathrm{meV}$ & \\
\hline$\pi p$ & $+7.116 \pm 0.011$ & $0.785 \pm 0.027$ & - & {$[6]$} \\
$\pi d$ & $-2.469 \pm 0.055$ & $1.095 \pm 0.129$ & - & {$[11,12]$} \\
$\pi t$ & & & & no data \\
$\pi^{3} \mathrm{He}$ & $+32.0 \pm 3.0$ & $28.0 \pm 7.0$ & $0.7-1.6$ & {$[20,22]$} \\
$\pi^{4} \mathrm{He}$ & $-75.7 \pm 2.0$ & $45.0 \pm 3.0$ & $0.7-2.2$ & {$[19,20,21,22]$} \\
\hline
\end{tabular}

\subsection{High-lying atomic $p$ states and quadrupole effects}

The resolution of the crystal spectrometer allows the separation of transitions to the states of different angular momenta for a given $n \leftrightarrow n^{\prime}$ transition (parallel transitions). Figure 3 shows for the case of $\pi N$ the $(5 f-4 d)$ and the $(5 d-4 p)$ lines besides the dominating circular transition $(5 g-4 f)$. The $4 p$ state is already affected by strong interaction and both shift and broadening were determined [23]. The values were found to be consistent with geometrical scaling applied to the results for the $2 p$ state [24]. In dedicated experiments, e. g., $4 p$ state effects could be studied systematically for nuclei from carbon to neon, elements which are available as pure elements or compounds. Isotopes are identified easily because of the good energy resolution. In favorable cases enriched targets allow in addition the determination of isotope effects.

In pionic atoms a contribution to shift and level splitting from strong interaction occurs along with the electromagnetic hyperfine splitting for $J \geq 1$ nuclei [25, 26]. Quadrupole effects reach up $40 \%$ of the size of the monopole contribution. In medium $Z$ atoms these 


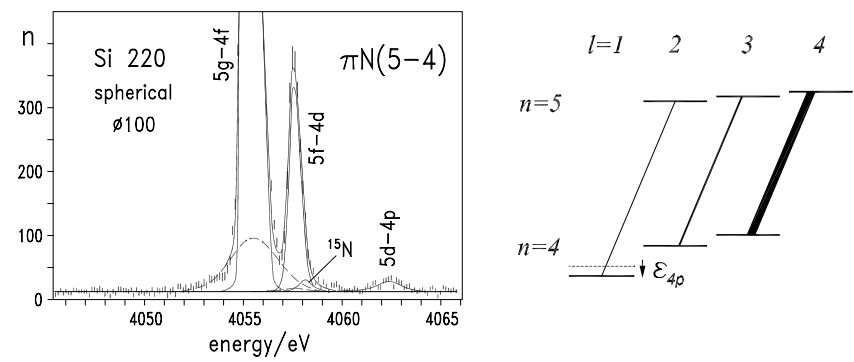

Figure 3: Parallel transitions of $\pi N(5-4)$ (from [23]. Bound-state QED may be tested from the energy difference of the circular $(5 g-4 f)$ and the first $(5 f-4 d)$ transition. The $4 p$ state is affected by strong interaction.

effects are hardly studied.

\section{Calibration standards}

\subsection{Pion mass}

For medium $Z$ nuclei, nuclear size and, hence, strong-interaction effects, are negligible in the intermediate part of cascade. Binding energies of the atomic levels can be calculated by the methods of QED with a precision better than $1 \mathrm{meV}[16]$. One application is the determination of the charged pion mass, where the wave length of the $\mu O(5 g-$ $4 f)$ transition $(4032 \mathrm{eV})$ is used as calibration standard for the $\pi N(5 g-4 f)$ transition $(4055 \mathrm{eV})$ in a simultaneous measurement using an $N_{2} / O_{2}$ gas mixture. An accuracy of about $1.5 \mathrm{ppm}$ in the mass determination is achievable by this method [17]. Two facts basically limit the accuracy of this approach: (i) statistics due the low stop rate for muons and (ii) the line broadening originating from Coulomb explosion (see chap. 4.3.2).

A possibility for further improvement is to replace the muonic calibration standard. Unfortunately, no strong pionic transition close in energy is available. However, a calibration chain involving fluorescence $\mathrm{X}$-rays and X-rays from few-electron atoms may be used to bridge the gap to a suitable pionic line [27] (Fig. 4 and 7). Energies of heliumor hydrogen-like electronic systems can be calculated with high precision from QED. They can be produced at high rate in electron-cyclotron resonance ion traps [28]. When antiprotonic atoms will be included, a copious set of calibration lines is available.

\subsection{Klein-Gordon equation}

Bound-state QED tests with muons have been performed from the beginning of exoticatom experiments. In the case of bosons, two precision measurements have been performed, which are in agreement with QED calculations [23, 29]. Though there is no 

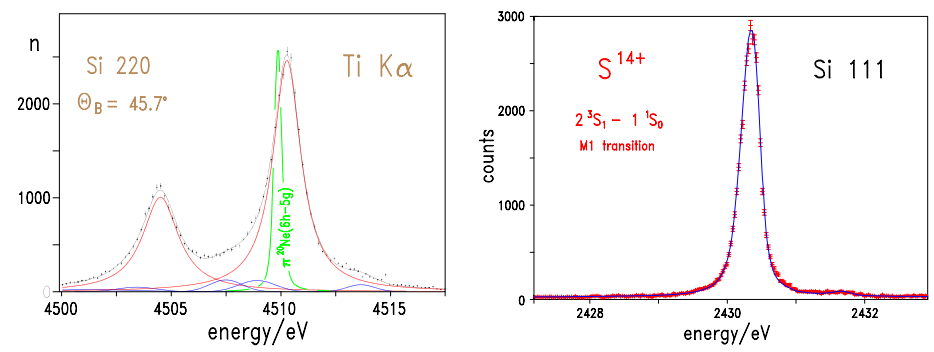

Figure 4: Left - Energy calibration of Ti K $\alpha$ lines by means of the $\pi N e(6-5)$ (from [27]). Right - Narrow M1 transition in helium-like sulphur which is used to determine the spectrometer resolution with utmost statistics.

reason to believe, that new physics may be found at the ppm level, which is the domain of X-ray spectroscopy, it is of outstanding importance to test and verify the methods of calculations [30]. A notorious example is the hyperfine splitting in antiprotonic deuterium, which led to a considerable confusion when compared to experiment (for details see $[1,31])$. In order to meet the accuracy achieved in the calculations, a determination of the line splitting from spectra like $\pi N$ or $\pi N e$ (Fig. 3 and 7 ) to better than one meV is desirable.

\section{Atomic cascade}

It became generally accepted that the formation of an exotic atom occurs by capture at the outmost electrons [32]. Hence, as a rule of thumb, the quantum cascade of the pion starts at about the radius of the outmost electron shell (Fig. 1). I. e., in the beginning the pion is attached to a screened nuclear charge forming even molecular states when coupled to a core of still bound nuclei.

In the case of the electrically neutral exotic hydrogen and to a lesser extent for helium, de-excitation is governed by collisional processes. When crossing other target molecules, fast transitions between angular momentum states $\left(n_{\pi}, \ell, m\right) \leftrightarrow\left(n_{\pi}, \ell \pm 1, m\right)$ occur because of the Coulomb field (Stark-mixing). Induced $s$ (and $p$ waves in $\pi H e$ ) lead to nuclear reactions and the X-ray cascade is substantially depleted. In $Z>2$ nuclei, Stark mixing does not play any role because of the short cascade time and the ion's charge. Electrons are quickly removed by Auger emission. At $n_{\pi} \cong 5$ radiative rates increase over Auger electron emission and the $\mathrm{X}$-ray cascade develops.

\subsection{Capture and de-excitation to high-lying states}

$\mathrm{X}$-ray intensities of the transitions between lower-lying states yield important constraints on capture. High-resolution spectroscopy can provide additional understanding by measuring (i) the relative population the high-lying $n_{\pi}, \ell=1$ states in the hydrogen isotopes 
or (ii) of the angular momentum states for a given $n_{\pi}$ for medium $Z$ atoms.

Cascade calculations for $\pi H$ predict very low intensities of $\left(n_{\pi}, \ell=1\right) \rightarrow 1 s$ transitions for $n_{\pi} \geq 6$ [33]. Crystal spectrometer measurements can resolve transitions up to initial states $\left(n_{\pi}=25, \ell=1\right)$.

As examples for case (ii), the parallel transitions in noble gases (Fig. 2: $\pi N e(7-6)$, and molecules (Fig. 3: $\pi N(5-4))$ may be compared. In favorable cases $\Delta n_{\pi}=1$ and $\Delta n_{\pi}=2$ transitions are measured simultaneously (Fig. 6). Combined with the detailed measurements of $\Delta n=1$ transitions performed with semiconductor detectors [34], a refined picture of the cascade is obtained. The effect of electron refilling, parametrised usually by an occupation number of electron shells, can be studied systematically.

\subsection{Interaction with the remaining electron shell}

De-excitation by electron emission is not restricted to the loosly bound electrons. In particular, when the outmost shell is depleted and the energy gain of $\Delta n_{\pi}=1$ transitions does not allow further ionisation, $\Delta n_{\pi} \gg 1$ Auger transitions may compete with radiative de-excitation leading to emission of 1 s electrons. The refilling of $\mathrm{K}$ holes from remaining electrons has been observed in antiprotonic atoms [35]. These fluorescence lines partly can be interpreted as the interplay of the antiproton at a certain degree of de-excitation and the presence of electrons (Fig. 5).

Owing to its much larger mass, the antiproton cascade starts at higher principle quantum numbers. Therefore, more steps are available for the depletion of the electron shells and a more circular cascade develops. Observation of electronic $\mathrm{X}$-rays in heavy muonic atoms was reported [36]. The study of low and medium $Z$ nuclei, in particular with better resolution, is still missing for pionic and muonic atoms.
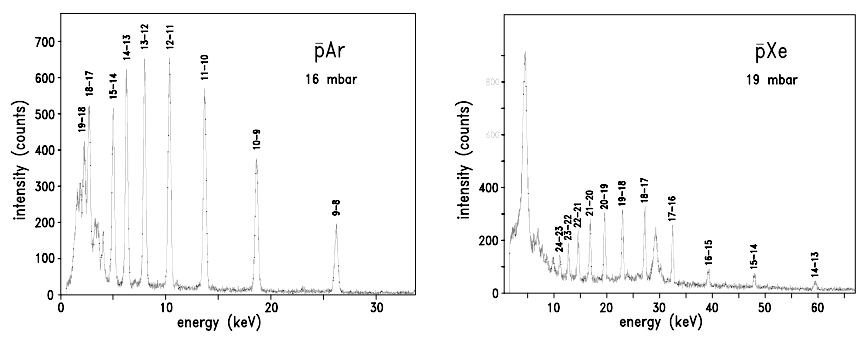

Figure 5: X-ray de-excitation chain in antiprotonic argon and xenon. A reduction of the line yields is observed when Auger emission becomes becomes possible $\left(n_{\bar{p}}=16\right)$. Strong electronic transitions are observed in xenon which originate from refilling of $\mathrm{L}$ and $\mathrm{K}$ holes produced during the de-excitation of the $\overline{\mathrm{p} X e}$ atom (from [35]). 


\subsection{Molecular effects}

\subsubsection{Pionic hydrogen isotopes at high density}

The molecular formation is well established in muon-catalysed fusion. Similarly, the formation of complexes like $\left[\left(\pi^{-} p p\right) \cdot p\right] e e$ has to be considered in the case of pionic hydrogen (see chap. 2.1). Possible radiative decay of the bound excited $\pi p$ atom causes satellite transitions affecting the determination of the strong-interaction shift [6]. No density dependence of the transition energy was observed and, hence, radiative decay assumed to be negligible.

In the case of liquid hydrogen, a weak evidence for a satellite transition in the low-energy tail is found by the numerical treatment, which, however, needs clarification (Fig. 6). Theoretical calculations suggest, that radiative decay may contribute significantly for deuterium and even dominate for tritium [10]. A search for such satellite transitions should be started first in deuterium.
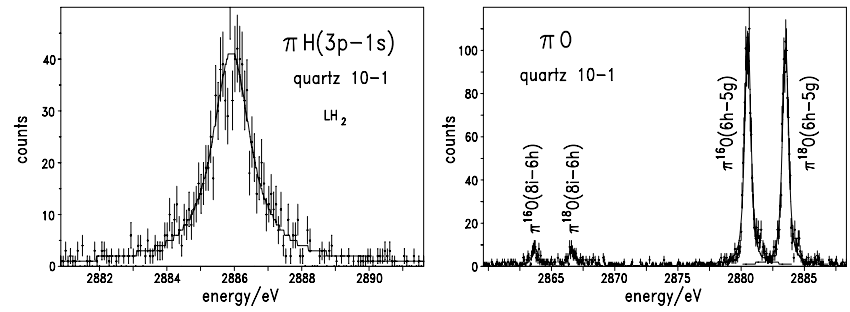

Figure 6: $\pi H(3 p-1 s)$ transition measured at liquid hydrogen density and $\pi O$ calibration lines (from [18]).

\subsubsection{Coulomb explosion}

Exotic atoms when formed from gaseous compounds are at first bound in molecular states. De-excitation starts by Auger emission of the electrons being in common to the constituents acting at the femtosecond time scale. Therefore, the ions experience a repulsive Coulomb force being still at a distance of about the original molecular bond length. The heavy particle finally will be attached to one of the ions continuing its de-excitation by Auger and X-ray emission.

The Coulomb repulsion leads to an acceleration and, consequently, a Doppler broadening of X-rays. First evidence was found from a comparison of the pressure dependence of the $\mathrm{K}$ line yields from muonic noble gases like neon with those from molecular targets like $\mathrm{N}_{2}$ or $\mathrm{O}_{2}$ [37]. The line yields of the atoms formed from molecules saturate at densities being one order of magnitude smaller, which was interpreted as enhanced electron refilling due to the velocity gain of the $\mu N$ or $\mu O$ atom. The direct observation of the Doppler broadening in exotic atoms was achieved by measuring the line widths of $\mu N$ or $\mu O$ lines 
using a crystal spectrometer (Fig. 7). It turned out that the line width is dominated by the Doppler broadening [38]. The line broadening yields the product of the ions' charge state and was found to be consistent with the number of the binding electrons. Coulomb
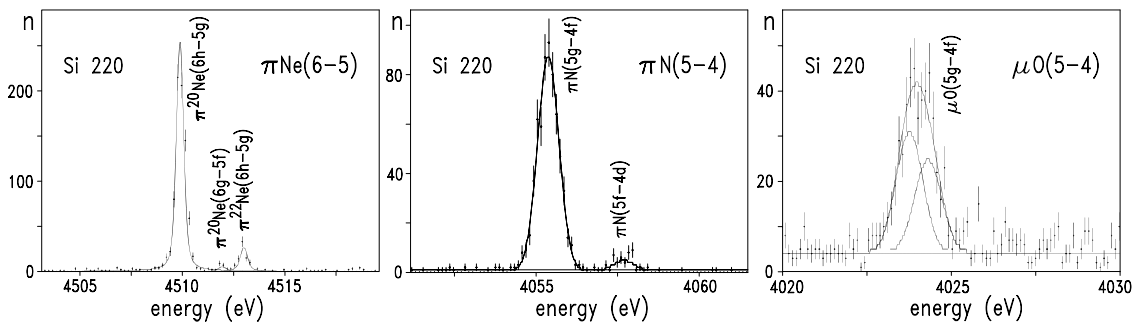

Figure 7: Direct observation of Coulomb explosion from the Doppler broadening (from $[38]$ ).

explosion is also observed after ion impact, leading to an immediate ionisation, as well after laser radiation, where ionisation can be adjusted to be very slow allowing to measure the bond length of the weakly ionised states. Due to the fast Auger emission, the exotic atom case should be more similar to ion bombardment. However, it seems that here exactly the binding electrons are affected. For binary molecules the effect is expected to be largest. It should decrease with increasing number of constituents and increasing ionicity of the binding, because here less elctrons are shared. X-ray spectroscopy of selected targets therefore probes features of molecular capture.

\section{Outlook}

The techniques worked out for the ongoing precision X-ray measurements of light exotic atoms allow a thorough study of hadronic, atomic and molecular effects. A continuation and further developments are highly desirable especially in view of the planned low-energy and high flux antiproton facilities.

\section{References}

[1] D. Gotta, Prog. Part. Nucl. Phys. 52, 133 (2004).

[2] T. S. Jensen and V. E. Markushin, Eur. Phys. J. D 19 (2002) 165; Eur. Phys. J. D 21 (2002) 261; Eur. Phys. J. D 21 (2002) 271.

[3] T. S. Jensen, contribution to this workshop.

[4] PSI experiment R-97.02.

[5] PSI experiment R-98.01, http://pihydrogen.web.psi.ch.

[6] L. M. Simons, contribution to this workshop. 
[7] T. E. O. Ericson, B. Loiseau and A. W. Thomas, Phys. Rev. C 66 (2002) 014005.

[8] U.-G. Meißner, U. Raha, and A. Rusetski, Eur. Phys. J. C 41, (2005) 213.

[9] E. Lindroth, J. Wallenius, and S. Jonsell, Phys. Rev. A 68 (2003) 032502.

[10] S. Kilic, J.-P. Karr, and L. Hilico, Phys. Rev. A 70 (2004) 250604.

[11] D. Chatellard et al., Nucl. Phys. A 625 (1997) 855.

[12] P. Hauser et al., Phys. Rev. C 58 (1998) R1869.

[13] V. Baru, J. Haidenbauer, C. Hanhart, and J. A. Niskanen, Eur. Phys. J. A 16 (2003) 437.

[14] D. Gotta et al., Phys. Rev. C 51 (1995) 469.

[15] E. Daum et al., Nucl. Phys. A 589 (1995) 553.

[16] P. Indelicato, priv. comm.

[17] D. F.Anagnostopoulos et al., Acta Phys. Pol. B 31 (2000) 2219.

[18] M. Hennebach, thesis, University of Cologne (2003), unpublished.

[19] G. Backenstoss et al., Nucl. Phys. A 232 (1974) 519.

[20] I. Schwanner et al., Nucl. Phys. A 412 (1984) 253.

[21] R. Landua and E. Klempt, Phys. Rev. Lett. 48 (1982) 1722.

[22] R. el Hassani, thesis, ETH Zürich no. 9256 (1990), unpublished.

[23] S. Lenz et al., Phys. Lett. B 416 (1998) 50.

[24] G. de Chambrier et al., Nucl. Phys. A 442 (1985) 637.

[25] F. Scheck, Nucl. Phys. B 43 (1972) 573.

[26] C. J.Batty, Nucl. Phys. A 335 (1981) 383, and references therein.

[27] D. F. Anagnostopoulos et al., Phys. Rev. Lett. 91 (2003) 240801.

[28] D. F. Anagnostopoulos et al., to be published in Nucl. Instr. Meth. A

[29] L. Delker et al., Phys. Rev. Lett. 42 (1979) 89.

[30] L. M. Simons et al., Proc. Int. Workshop on Exotic Atoms (EXA02), Vienna, 2002, (Austrian Academy of Science Press, Vienna, 2003), p. 197.

[31] D. Gotta et al., Nucl. Phys. A 660 (1999) 283.

[32] F. J. Hartmann, Proc. Int. School of Physics of Exotic Atoms, eds. L. M. Simons, D. Horváth and G. Torelli, Erice, Italy, 1989, (Plenum Press, New York, 1990), p. 127, and references therein.

[33] T. Jensen, priv. comm.

[34] K. Kirch et al., Phys. Rev. A 59 (1999) 3375.

[35] K. Rashid et al., in preparation.

[36] W. D. Fromm et al., Phys. Lett., 55 B (1975) 377.

[37] P. Ehrhart, F. J. Hartmann, E. Köhler, and H. Daniel, Z. Phys. A 311 (1983) 259.

[38] T. Siems et al., Phys. Rev. Lett. 84 (2000) 4573. 